\title{
MAsses AND DECAY CONSTANTS OF THE HEAVy TENSOR MESONS WITH QCD SUM RULES
}

\author{
Zhi-Gang Wang 1, Zun-Yan Di \\ Department of Physics, North China Electric Power University, Baoding 071003, P. R. China
}

\begin{abstract}
In this article, we calculate the contributions of the vacuum condensates up to dimension- 6 in the operator product expansion, study the masses and decay constants of the heavy tensor mesons $D_{2}^{*}(2460), D_{s 2}^{*}(2573), B_{2}^{*}(5747), B_{s 2}^{*}(5840)$ using the QCD sum rules. The predicted masses are in excellent agreement with the experimental data, while the ratios of the decay constants $\left.\frac{f_{D_{s 2}^{*}}}{f_{D_{2}^{*}}} \approx \frac{f_{B_{s 2}^{*}}}{f_{B_{2}^{*}}} \approx \frac{f_{D_{s}}}{f_{D}}\right|_{\exp }$, where the exp denotes the experimental value.
\end{abstract}

PACS number: 13.20.Fc, 13.20.He

Key words: Decay constants, Tensor mesons, QCD sum rules

\section{Introduction}

In recent years, the Babar, Belle, CLEO, CDF, D0, LHCb and BESIII collaborations have observed (or confirmed) many charmonium-like states and revitalized the interest in the spectroscopy of the charmonium states [1. There are also some progresses in the spectroscopy of the conventional heavy mesons, several excited states have been observed, such as the $D_{s 1}^{*}(2700), D_{s J}^{*}(2860), D_{s J}(3040)$, $D_{J}(2580), D_{J}^{*}(2650), D_{J}(2740), D_{J}^{*}(2760), D_{J}(3000), D_{J}^{*}(3000), B_{1}(5721), B_{2}^{*}(5747), B_{s 1}(5830)$, $B_{s 2}^{*}(5840), B(5970)$, etc [2]. In 2007, the D0 collaboration firstly observed the $B_{1}(5721)^{0}$ and $B_{2}(5747)^{0}$ [3], later the CDF collaboration confirmed them [4. Also in 2007, the CDF collaboration observed the $B_{s 1}(5830)$ and $B_{s 2}^{*}(5840)$ [5]. The D0 collaboration confirmed the $B_{s 2}^{*}(5840)$ [6]. In 2012 , the LHCb collaboration updated the masses $M_{B_{s 1}}=(5828.40 \pm 0.04 \pm 0.04 \pm 0.41) \mathrm{MeV}$ and $M_{B_{s 2}^{*}}=(5839.99 \pm 0.05 \pm 0.11 \pm 0.17) \mathrm{MeV}$ [7]. The heavy-light mesons can be classified in doublets according to the total angular momentum of the light antiquark $\vec{s}_{\ell}, \vec{s}_{\ell}=\vec{s}_{\bar{q}}+\vec{L}$, where the $\vec{s}_{\bar{q}}$ and $\vec{L}$ are the spin and orbital angular momentum of the light antiquark, respectively. Now the $J_{s_{\ell}}^{P}=$ $\left(0^{+}, 1^{+}\right)_{\frac{1}{2}}$ doublets $\left(D_{0}^{*}(2400), D_{1}^{\prime}(2430)\right),\left(D_{s 0}^{*}(2317), D_{s 1}^{\prime}(2460)\right)$, the $J_{s_{\ell}}^{P}=\left(1^{+}, 2^{+}\right)_{\frac{3}{2}}$ doublets $\left(D_{1}(2420), D_{2}^{*}(2460)\right),\left(D_{s 1}(2536), D_{s 2}^{*}(2573)\right),\left(B_{1}(5721), B_{2}^{*}(5747)\right),\left(B_{s 1}(5830), B_{s 2}^{*}(5840)\right)$ are complete [2].

The QCD sum rules is a powerful nonperturbative theoretical tool in studying the ground state hadrons [8, 9, 10, 11]. In the QCD sum rules, the operator product expansion is used to expand the time-ordered currents into a series of quark and gluon condensates which parameterize the long distance properties. We can obtain copious information on the hadronic parameters at the phenomenological side by taking the quark-hadron duality [8, 9, 10, 11. There have been many works on the $J_{s_{\ell}}^{P}=\left(0^{-}, 1^{-}\right)_{\frac{1}{2}}$ doublets with the full QCD sum rules [10, 11] (or Ref. [12] for recent works), while the works on the $J_{s_{\ell}}^{P}=\left(0^{+}, 1^{+}\right)_{\frac{1}{2}}$ and $\left(1^{+}, 2^{+}\right)_{\frac{3}{2}}$ doublets are few [13, 14, 15. For the works on the QCD sum rules combined with the heavy quark effective theory, one can consult Refs. 16, 17. In Ref. 14, H. Sundu et al study the masses and decay constants of the tensor mesons $D_{2}^{*}(2460)$ and $D_{s 2}^{*}(2573)$ with the QCD sum rules by taking into account the perturbative terms and the mixed condensates in the operator product expansion, the contributions of the gluon condensate, three-gluon condensate and four-quark condensate are neglected. Neglecting the vacuum condensates of dimension- 4 and 6 impairs the predictive ability.

In this article, we calculate the contributions of the vacuum condensates up to dimension- 6 in the operator product expansion consistently, study the masses and decay constants of the heavy tensor mesons $D_{2}^{*}(2460), D_{s 2}^{*}(2573), B_{2}^{*}(5747), B_{s 2}^{*}(5840)$ with the QCD sum rules.

\footnotetext{
${ }^{1}$ E-mail,zgwang@aliyun.com.
} 
The article is arranged as follows: we derive the QCD sum rules for the masses and decay constants of the heavy tensor mesons in Sect.2; in Sect.3, we present the numerical results and discussions; and Sect.4 is reserved for our conclusions.

\section{QCD sum rules for the heavy tensor mesons}

In the following, we write down the two-point correlation functions $\Pi_{\mu \nu \alpha \beta}(p)$ in the QCD sum rules,

$$
\begin{aligned}
\Pi_{\mu \nu \alpha \beta}(p) & =\left.i \int d^{4} x e^{i p \cdot(x-y)}\left\langle 0\left|T\left\{J_{\mu \nu}(x) J_{\alpha \beta}^{\dagger}(y)\right\}\right| 0\right\rangle\right|_{y=0} \\
J_{\mu \nu}(x) & =i \bar{Q}(x)\left(\gamma_{\mu} \overleftrightarrow{\partial}_{\nu}+\gamma_{\nu} \overleftrightarrow{\partial}_{\mu}-\frac{2}{3} \widetilde{g}_{\mu \nu} \stackrel{\leftrightarrow}{\partial}\right) q(x) \\
\overleftrightarrow{\partial}_{\mu} & =\vec{\partial}_{\mu}-\overleftarrow{\partial}_{\mu} \\
\widetilde{g}_{\mu \nu} & =g_{\mu \nu}-\frac{p_{\mu} p_{\nu}}{p^{2}}
\end{aligned}
$$

where $Q=c, b$ and $q=u, d, s$, the tensor currents $J_{\mu \nu}(x)$ interpolate the heavy tensor mesons $D_{2}^{*}(2460), D_{s 2}^{*}(2573), B_{2}^{*}(5747)$ and $B_{s 2}^{*}(5840)$, respectively. In Ref.[18], Aliev and Shifman take the tensor currents $\eta_{\mu \nu}^{1}(x)$,

$$
\eta_{\mu \nu}^{1}(x)=\frac{1}{2} i \bar{q}(x)\left(\gamma_{\mu} \stackrel{\leftrightarrow}{D}_{\nu}+\gamma_{\nu} \stackrel{\leftrightarrow}{D}_{\mu}\right) q(x),
$$

with $D_{\mu}=\partial_{\mu}-i g_{s} G_{\mu}$ and the $G_{\mu}$ is the gluon field, to study the light tensor mesons. Later, Reinders, Yazaki, Rubinstein take the tensor currents $\eta_{\mu \nu}^{2}(x)$,

$$
\eta_{\mu \nu}^{2}(x)=i \bar{q}(x)\left(\gamma_{\mu} \stackrel{\leftrightarrow}{\partial}_{\nu}+\gamma_{\nu} \stackrel{\leftrightarrow}{\partial}_{\mu}-\frac{2}{3} \widetilde{g}_{\mu \nu} \stackrel{\leftrightarrow}{\not}\right) q(x)
$$

to study the light tensor mesons [19]. In Ref. [20], Bagan and Narison restudy the light tensor mesons with the tensor currents $\eta_{\mu \nu}^{1}(x)$. For recent works on the light tensor mesons with the QCD sum rules, see Ref. 21. We have two choice, i.e. we can choose either the partial derivative $\partial_{\mu}$ or the covariant derivative $D_{\mu}$ in constructing the interpolating currents. The currents $\eta_{\mu \nu}^{1}(x)$ with the covariant derivative $D_{\mu}$ are gauge invariant, but blur the physical interpretation of the $\stackrel{\leftrightarrow}{D}_{\mu}$ as the angular momentum; on the other hand, the currents $\eta_{\mu \nu}^{2}(x)$ with the partial derivative $\partial_{\mu}$ are not gauge invariant, but manifest the physical interpretation of the $\stackrel{\leftrightarrow}{\partial}_{\mu}$ as the angular momentum. In this article, we will present the results come from the currents with both the partial derivative and the covariant derivative.

We can insert a complete set of intermediate hadronic states with the same quantum numbers as the current operators $J_{\mu \nu}(x)$ into the correlation functions $\Pi_{\mu \nu \alpha \beta}(p)$ to obtain the hadronic representation [8, 9. After isolating the ground state contributions from the heavy tensor mesons, we get the following result,

$$
\begin{aligned}
\Pi_{\mu \nu \alpha \beta}(p) & =\frac{f_{T}^{2} M_{T}^{4}}{M_{T}^{2}-p^{2}} \mathrm{P}_{\mu \nu \alpha \beta}+\cdots \\
& =\Pi\left(p^{2}\right) \mathrm{P}_{\mu \nu \alpha \beta}
\end{aligned}
$$

where the decay constants $f_{T}$ are defined by

$$
\left\langle 0\left|J_{\mu \nu}(0)\right| T(p)\right\rangle=f_{T} M_{T}^{2} \varepsilon_{\mu \nu},
$$


the $\varepsilon_{\mu \nu}$ are the polarization vectors of the tensor mesons with the following properties [22],

$$
\begin{aligned}
\mathrm{P}_{\mu \nu \alpha \beta} & =\sum_{\lambda} \varepsilon_{\mu \nu}^{*}(\lambda, p) \varepsilon_{\alpha \beta}(\lambda, p)=\frac{\widetilde{g}_{\mu \alpha} \widetilde{g}_{\nu \beta}+\widetilde{g}_{\mu \beta} \widetilde{g}_{\nu \alpha}}{2}-\frac{\widetilde{g}_{\mu \nu} \widetilde{g}_{\alpha \beta}}{3} \\
p^{\mu} \mathrm{P}_{\mu \nu \alpha \beta} & =\widetilde{g}^{\mu \nu} \mathrm{P}_{\mu \nu \alpha \beta}=\widetilde{g}^{\alpha \beta} \mathrm{P}_{\mu \nu \alpha \beta}=g^{\mu \nu} \mathrm{P}_{\mu \nu \alpha \beta}=g^{\alpha \beta} \mathrm{P}_{\mu \nu \alpha \beta}=0 \\
5 & =\mathrm{P}_{\mu \nu \alpha \beta} \mathrm{P}^{\mu \nu \alpha \beta} .
\end{aligned}
$$

Now, we briefly outline the operator product expansion for the correlation functions $\Pi_{\mu \nu \alpha \beta}(p)$ in perturbative QCD. We contract the quark fields in the correlation functions $\Pi_{\mu \nu \alpha \beta}(p)$ with Wick theorem firstly,

$$
\begin{aligned}
\Pi\left(p^{2}\right) & =\frac{1}{5} \mathrm{P}^{\mu \nu \alpha \beta} \Pi_{\mu \nu \alpha \beta}(p) \\
& =-\left.\frac{i}{5} \mathrm{P}^{\mu \nu \alpha \beta} \int d^{4} x e^{i p \cdot(x-y)} \operatorname{Tr}\left\{\Gamma_{\mu \nu} S_{i j}(x-y) \Gamma_{\alpha \beta} S_{j i}^{Q}(y-x)\right\}\right|_{y=0}
\end{aligned}
$$

where

$$
\begin{aligned}
& \Gamma_{\mu \nu}=i\left(\gamma_{\mu} \frac{\stackrel{\leftrightarrow}{\partial}}{\partial x^{\nu}}+\gamma_{\nu} \frac{\stackrel{\leftrightarrow}{\partial}}{\partial x^{\mu}}-\frac{2}{3} \widetilde{g}_{\mu \nu} \gamma^{\tau} \frac{\stackrel{\leftrightarrow}{\partial}}{\partial x^{\tau}}\right) \\
& \Gamma_{\alpha \beta}=i\left(\gamma_{\alpha} \frac{\stackrel{\leftrightarrow}{\partial}}{\partial y^{\beta}}+\gamma_{\beta} \frac{\stackrel{\leftrightarrow}{\partial}}{\partial y^{\alpha}}-\frac{2}{3} \widetilde{g}_{\alpha \beta} \gamma^{\tau} \frac{\stackrel{\leftrightarrow}{\partial}}{\partial y^{\tau}}\right), \\
& S_{i j}(x)=\frac{i \delta_{i j} \not x}{2 \pi^{2} x^{4}}-\frac{\delta_{i j} m_{q}}{4 \pi^{2} x^{2}}-\frac{\delta_{i j}}{12}\langle\bar{q} q\rangle+\frac{i \delta_{i j} \not x m_{q}\langle\bar{q} q\rangle}{48}-\frac{\delta_{i j} x^{2}\left\langle\bar{q} g_{s} \sigma G q\right\rangle}{192}+\frac{i \delta_{i j} x^{2} \not x m_{q}\left\langle\bar{q} g_{s} \sigma G q\right\rangle}{1152} \\
& -\frac{i g_{s} G_{\alpha \beta}^{a} t_{i j}^{a}\left(\not \partial \sigma^{\alpha \beta}+\sigma^{\alpha \beta} \not x\right)}{32 \pi^{2} x^{2}}+\frac{i \delta_{i j} x^{2} \not \not x g_{s}^{2}\left\langle\bar{q} \gamma_{\mu} t^{n} q \bar{q} \gamma^{\mu} t^{n} q\right\rangle}{3456}-\frac{1}{8}\left\langle\bar{q}_{j} \sigma^{\mu \nu} q_{i}\right\rangle \sigma_{\mu \nu} \\
& -\frac{1}{4}\left\langle\bar{q}_{j} \gamma^{\mu} q_{i}\right\rangle \gamma_{\mu}+\cdots \\
& S_{i j}^{Q}(x)=\frac{i}{(2 \pi)^{4}} \int d^{4} k e^{-i k \cdot x}\left\{\frac{\delta_{i j}}{\not k-m_{Q}}-\frac{g_{s} G_{\alpha \beta}^{n} t_{i j}^{n}}{4} \frac{\sigma^{\alpha \beta}\left(\not k+m_{Q}\right)+\left(\not k+m_{Q}\right) \sigma^{\alpha \beta}}{\left(k^{2}-m_{Q}^{2}\right)^{2}}\right. \\
& +\frac{g_{s} D_{\alpha} G_{\beta \lambda}^{n} t_{i j}^{n}\left(f^{\lambda \beta \alpha}+f^{\lambda \alpha \beta}\right)}{3\left(k^{2}-m_{Q}^{2}\right)^{4}}-\frac{g_{s}^{2}\left(t^{a} t^{b}\right)_{i j} G_{\alpha \beta}^{a} G_{\mu \nu}^{b}\left(f^{\alpha \beta \mu \nu}+f^{\alpha \mu \beta \nu}+f^{\alpha \mu \nu \beta}\right)}{4\left(k^{2}-m_{Q}^{2}\right)^{5}} \\
& \left.+\frac{i\left\langle g_{s}^{3} G G G\right\rangle}{48} \frac{\left(k+m_{Q}\right)\left[k\left(k^{2}-3 m_{Q}^{2}\right)+2 m_{Q}\left(2 k^{2}-m_{Q}^{2}\right)\right]\left(\not k+m_{Q}\right)}{\left(k^{2}-m_{Q}^{2}\right)^{6}}+\cdots\right\}, \\
& f^{\lambda \alpha \beta}=\left(\not k+m_{Q}\right) \gamma^{\lambda}\left(\not k+m_{Q}\right) \gamma^{\alpha}\left(\not k+m_{Q}\right) \gamma^{\beta}\left(\not k+m_{Q}\right), \\
& f^{\alpha \beta \mu \nu}=\left(\not k+m_{Q}\right) \gamma^{\alpha}\left(\not k+m_{Q}\right) \gamma^{\beta}\left(\not k+m_{Q}\right) \gamma^{\mu}\left(\not k+m_{Q}\right) \gamma^{\nu}\left(\not k+m_{Q}\right) \text {, }
\end{aligned}
$$

$t^{n}=\frac{\lambda^{n}}{2}$, the $\lambda^{n}$ is the Gell-Mann matrix, the $i, j$ are color indexes(One can consult Refs. 29, 23] for the technical details in deriving the full heavy quark and light quark propagators, respectively.); then compute the integrals both in the coordinate and momentum spaces; finally obtain the QCD spectral density through dispersion relation,

$$
\Pi\left(p^{2}\right)=\frac{1}{\pi} \int_{m_{Q}^{2}}^{\infty} \frac{\operatorname{Im} \Pi(s)}{s-p^{2}}=\int_{m_{Q}^{2}}^{\infty} \frac{\rho_{Q C D}(s)}{s-p^{2}} .
$$



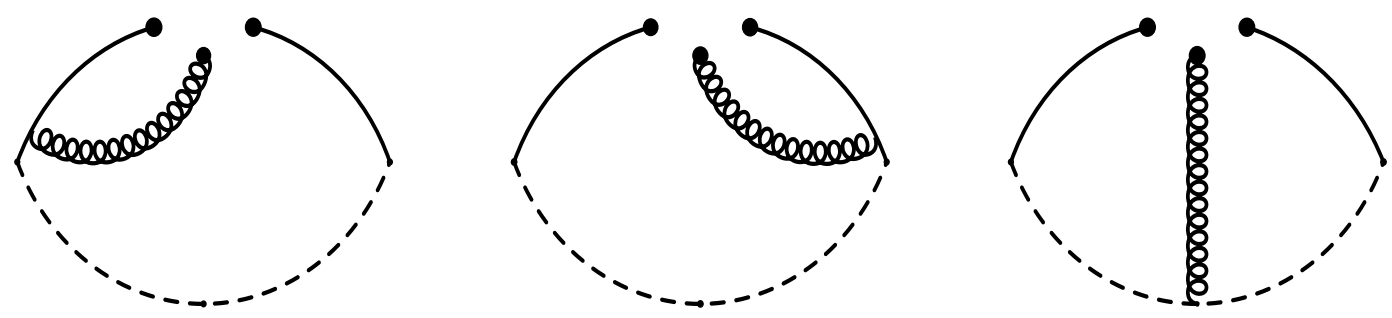

Figure 1: The diagrams contribute to the mixed condensate $\left\langle\bar{q} g_{s} \sigma G q\right\rangle$.
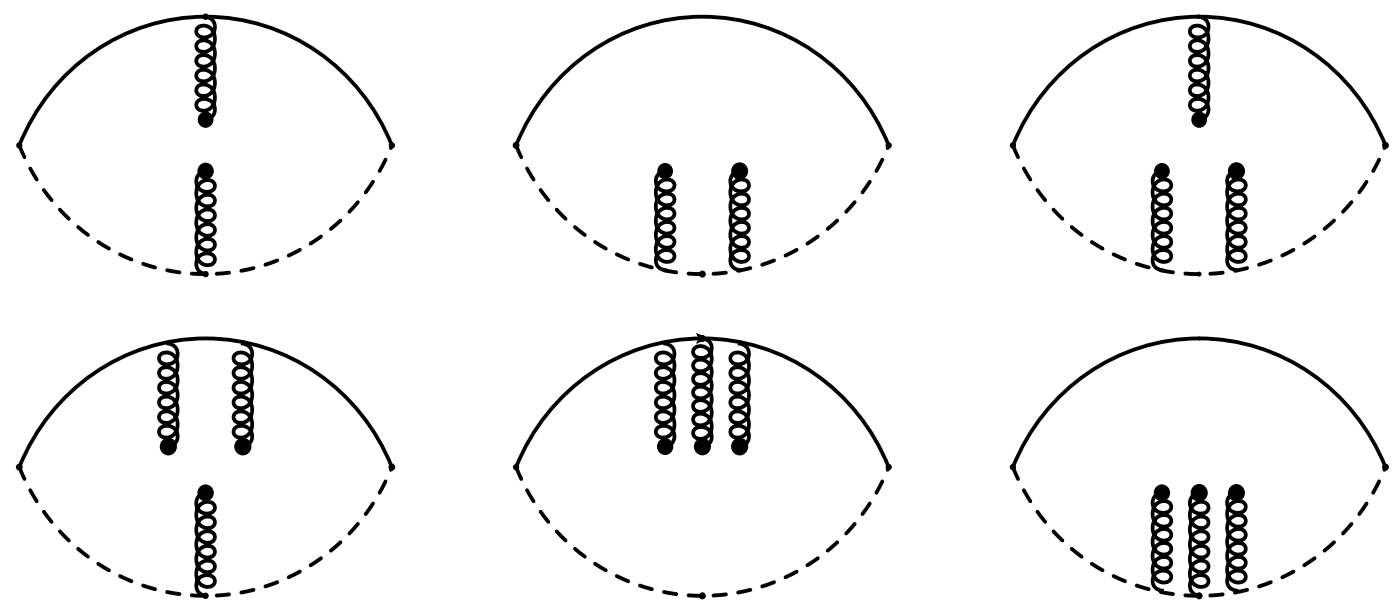

Figure 2: The diagrams contribute to the gluon condensate $\left\langle\frac{\alpha_{s} G G}{\pi}\right\rangle$ and three-gluon condensate $\left\langle g_{s}^{3} G G G\right\rangle$.
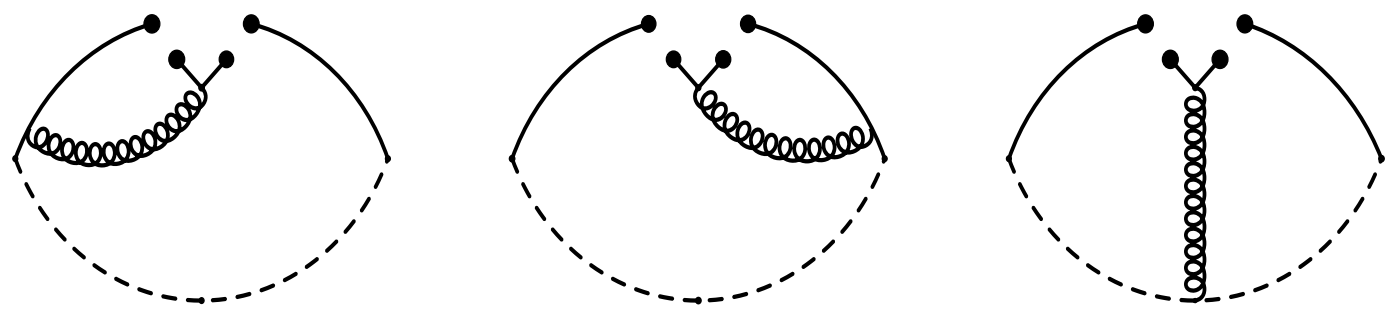

Figure 3: The diagrams contribute to the four-quark condensate $\langle\bar{q} q\rangle^{2}$. 
In Eq.(10), we retain the terms $\left\langle\bar{q}_{j} \sigma_{\mu \nu} q_{i}\right\rangle$ and $\left\langle\bar{q}_{j} \gamma_{\mu} q_{i}\right\rangle$ originate from the Fierz re-ordering of the $\left\langle q_{i} \bar{q}_{j}\right\rangle$ to absorb the gluons emitted from the heavy quark lines to form $\left\langle\bar{q}_{j} g_{s} G_{\alpha \beta}^{a} t_{m n}^{a} \sigma_{\mu \nu} q_{i}\right\rangle$ and $\left\langle\bar{q}_{j} \gamma_{\mu} q_{i} g_{s} D_{\nu} G_{\alpha \beta}^{a} t_{m n}^{a}\right\rangle$ so as to extract the mixed condensate and four-quark condensate $\left\langle\bar{q} g_{s} \sigma G q\right\rangle$ and $g_{s}^{2}\langle\bar{q} q\rangle^{2}$, respectively. In Ref.[14, such contributions are neglected.

We take quark-hadron duality below the continuum thresholds $s_{0}$ and perform the Borel transform with respect to the variable $P^{2}=-p^{2}$ to obtain the QCD sum rules,

$$
\begin{aligned}
f_{T}^{2} M_{T}^{4} \exp \left(-\frac{M_{T}^{2}}{M^{2}}\right)= & \frac{1}{10 \pi^{2}} \int_{m_{Q}^{2}}^{s_{0}} d s \frac{\left(s-m_{Q}^{2}\right)^{4}\left(3 s+2 m_{Q}^{2}\right)+10 m_{q} m_{Q} s\left(s-m_{Q}^{2}\right)^{3}}{s^{3}} \exp \left(-\frac{s}{M^{2}}\right) \\
& -\frac{m_{Q}\left\langle\bar{q} g_{s} \sigma G q\right\rangle}{2} \exp \left(-\frac{m_{Q}^{2}}{M^{2}}\right)+\left(\frac{m_{q}\left\langle\bar{q} g_{s} \sigma G q\right\rangle}{6}-\frac{2 g_{s}^{2}\langle\bar{q} q\rangle^{2}}{81}\right) \\
& \left(1+\frac{m_{Q}^{2}}{M^{2}}\right) \exp \left(-\frac{m_{Q}^{2}}{M^{2}}\right)+m_{Q}^{2}\left\langle\frac{\alpha_{s} G G}{\pi}\right\rangle\left[\left(\frac{1}{18}-\frac{2}{9} \frac{m_{Q}^{2}}{M^{2}}-\frac{5}{18} \frac{M^{2}}{m_{Q}^{2}}\right)\right. \\
& \left.\exp \left(-\frac{m_{Q}^{2}}{M^{2}}\right)+\left(\frac{1}{6} \frac{m_{Q}^{2}}{M^{2}}+\frac{2}{9} \frac{m_{Q}^{4}}{M^{4}}\right) \Gamma\left(0, \frac{m_{Q}^{2}}{M^{2}}\right)\right]+\frac{\left\langle g_{s}^{3} G G G\right\rangle}{48 \pi^{2}} \\
& {\left[\left(-\frac{20}{3}-\frac{16}{3} \frac{m_{Q}^{2}}{M^{2}}\right) \exp \left(-\frac{m_{Q}^{2}}{M^{2}}\right)+\left(12 \frac{m_{Q}^{2}}{M^{2}}+\frac{16}{3} \frac{m_{Q}^{4}}{M^{4}}\right) \Gamma\left(0, \frac{m_{Q}^{2}}{M^{2}}\right)\right] }
\end{aligned}
$$

$\Gamma(0, x)=\int_{0}^{\infty} d t \frac{1}{t} e^{-x t}$. In this article, we carry out the operator product expansion up to the vacuum condensates of dimension 6 in the leading order approximation. In Figs.1-3, we express the contributions of the mixed condensates, four-quark condensates, gluon condensates and threegluon condensates in terms of Feynman diagrams, which are drawn up directly from Eqs.(8-11). In the Feynman diagrams, we use the solid and dashed lines to represent the light and heavy quark propagators, respectively. The perturbative contributions are consistent with that obtained in Ref. 14] (also that in Refs. 18, 19, 20] in the limit $m_{Q} \rightarrow 0$ ), however, the contributions of the mixed condensates differ from that obtained in Ref.[14. In Ref. 14, the third Feynman diagram in Fig. 1 is neglected, the Feynman diagrams in Figs.2-4 are fully neglected. The contributions of the gluon condensate, three-gluon condensate and four-quark condensate are obtained originally in this work. As far as the contributions of the vacuum condensates are concerned, the light quark currents and the heavy-light quark currents lead to different expressions, which do not coincide in the limit $m_{Q} \rightarrow 0$.

If we replace the partial derivative with the covariant derivative in the interpolating currents, the following terms

$$
\begin{aligned}
& -\frac{M^{2}}{3}\left\langle\frac{\alpha_{s} G G}{\pi}\right\rangle \exp \left(-\frac{m_{Q}^{2}}{M^{2}}\right)-\frac{\left\langle g_{s}^{3} G G G\right\rangle}{24 \pi^{2}}\left(\frac{5}{6}+\frac{m_{Q}^{2}}{M^{2}}\right) \exp \left(-\frac{m_{Q}^{2}}{M^{2}}\right) \\
& +\frac{\left\langle g_{s}^{3} G G G\right\rangle}{24 \pi^{2}} \frac{m_{Q}^{2}}{M^{2}}\left(\frac{11}{6}+\frac{m_{Q}^{2}}{M^{2}}\right) \Gamma\left(0, \frac{m_{Q}^{2}}{M^{2}}\right),
\end{aligned}
$$

should be added to the right side of the Eq.(13). In the leading order approximation, the perturbative terms of the QCD spectral densities are not modified, we take into account the additional 

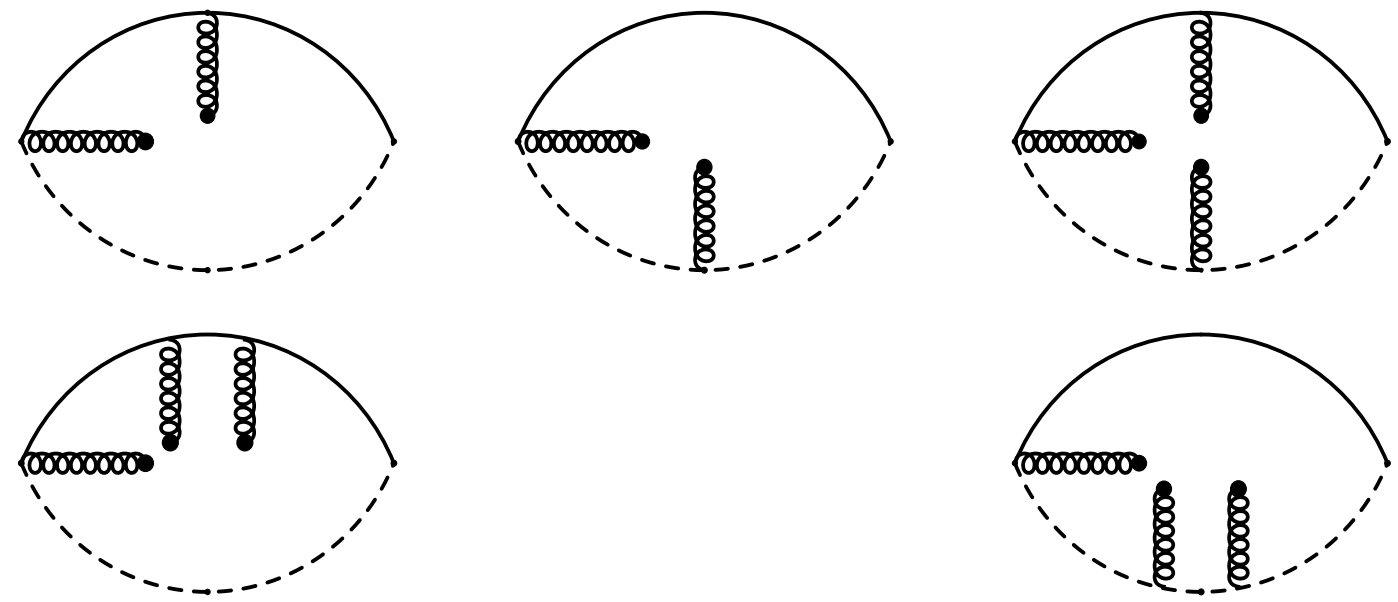

Figure 4: The additional diagrams contribute to the gluon condensate $\left\langle\frac{\alpha_{s} G G}{\pi}\right\rangle$ and three-gluon condensate $\left\langle g_{s}^{3} G G G\right\rangle$ from the covariant derivative.

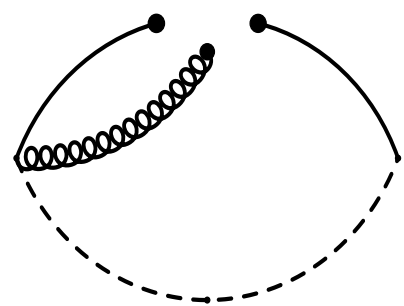

Figure 5: The additional diagram contributes to the mixed condensate $\left\langle\bar{q} g_{s} \sigma G q\right\rangle$ from the covariant derivative.

contributions by the modified vertexes,

$$
\begin{aligned}
& \Gamma_{\mu \nu}=i\left(\gamma_{\mu} \frac{\stackrel{\leftrightarrow}{\partial}}{\partial x^{\nu}}+\gamma_{\nu} \frac{\stackrel{\leftrightarrow}{\partial}}{\partial x^{\mu}}-\frac{2}{3} \widetilde{g}_{\mu \nu} \gamma^{\tau} \frac{\stackrel{\leftrightarrow}{\partial}}{\partial x^{\tau}}\right)+2 g_{s}\left(\gamma_{\mu} G_{\nu}(x)+\gamma_{\nu} G_{\mu}(x)-\frac{2}{3} \widetilde{g}_{\mu \nu} \gamma^{\tau} G_{\tau}(x)\right) \\
& \Gamma_{\alpha \beta}=i\left(\gamma_{\alpha} \frac{\stackrel{\leftrightarrow}{\partial}}{\partial y^{\beta}}+\gamma_{\beta} \frac{\stackrel{\leftrightarrow}{\partial}}{\partial y^{\alpha}}-\frac{2}{3} \widetilde{g}_{\alpha \beta} \gamma^{\tau} \frac{\stackrel{\leftrightarrow}{\partial}}{\partial y^{\tau}}\right)+2 g_{s}\left(\gamma_{\alpha} G_{\beta}(y)+\gamma_{\beta} G_{\alpha}(y)-\frac{2}{3} \widetilde{g}_{\alpha \beta} \gamma^{\tau} G_{\tau}(y)\right)
\end{aligned}
$$

where $G_{\mu}(x)=\frac{1}{2} x^{\theta} G_{\theta \mu}(0)+\cdots$ and $G_{\alpha}(y)=\frac{1}{2} y^{\theta} G_{\theta \alpha}(0)+\cdots=0$ in the fixed point gauge. The contributions are shown explicitly by the Feynman diagrams in Fig.4. There are no additional contributions come from the mixed quark condensate, i.e. we calculate the Feynman diagram in Fig.5 and observe that the contribution is zero.

Differentiate Eq.(13) with respect to $\frac{1}{M^{2}}$, then eliminate the decay constants $f_{T}$, we obtain the QCD sum rules for the masses of the tensor mesons. In this article, we take into account the contributions come from the covariant derivative.

\section{Numerical results and discussions}

The masses of the tensor mesons listed in the Review of Particle Physics are $M_{D_{2}^{*}(2460)^{ \pm}}=(2464.3 \pm$ 1.6) $\mathrm{MeV}, M_{D_{2}^{*}(2460)^{0}}=(2461.8 \pm 0.7) \mathrm{MeV}, M_{D_{s 2}^{*}(2573)}=(2571.9 \pm 0.8) \mathrm{MeV}, M_{B_{2}^{*}(5747)^{0}}=$ 
$(5743 \pm 5) \mathrm{MeV}, M_{B_{s 2}^{*}(5840)^{0}}=(5839.96 \pm 0.20) \mathrm{MeV}$ [2]. We can take the threshold parameters as $s_{D_{2}^{*}}^{0}=(8.5 \pm 0.5) \mathrm{GeV}^{2}, s_{D_{s 2}^{*}}^{0}=(9.5 \pm 0.5) \mathrm{GeV}^{2}, s_{B_{2}^{*}}^{0}=(39 \pm 1) \mathrm{GeV}^{2}, s_{B_{s 2}^{*}}^{0}=(41 \pm 1) \mathrm{GeV}^{2}$ tentatively to avoid the contaminations of the high resonances and continuum states, the energy gaps $\sqrt{s_{T}^{0}}-M_{T}=(0.4-0.6) \mathrm{GeV}$, the contributions of the ground states are fully included.

The quark condensates and mixed condensates are taken to be the standard values $\langle\bar{q} q\rangle=$ $-(0.24 \pm 0.01 \mathrm{GeV})^{3},\langle\bar{s} s\rangle=(0.8 \pm 0.1)\langle\bar{q} q\rangle,\left\langle\bar{q} g_{s} \sigma G q\right\rangle=m_{0}^{2}\langle\bar{q} q\rangle,\left\langle\bar{s} g_{s} \sigma G s\right\rangle=m_{0}^{2}\langle\bar{s} s\rangle, m_{0}^{2}=$ $(0.8 \pm 0.1) \mathrm{GeV}^{2}$ at the energy scale $\mu=1 \mathrm{GeV}$ [11]. The quark condensate and mixed quark condensate evolve with the renormalization group equation, $\langle\bar{q} q\rangle(\mu)=\langle\bar{q} q\rangle(Q)\left[\frac{\alpha_{s}(Q)}{\alpha_{s}(\mu)}\right]^{\frac{4}{9}},\langle\bar{s} s\rangle(\mu)=$ $\langle\bar{s} s\rangle(Q)\left[\frac{\alpha_{s}(Q)}{\alpha_{s}(\mu)}\right]^{\frac{4}{9}},\left\langle\bar{q} g_{s} \sigma G q\right\rangle(\mu)=\left\langle\bar{q} g_{s} \sigma G q\right\rangle(Q)\left[\frac{\alpha_{s}(Q)}{\alpha_{s}(\mu)}\right]^{\frac{2}{27}}$ and $\left\langle\bar{s} g_{s} \sigma G s\right\rangle(\mu)=\left\langle\bar{s} g_{s} \sigma G s\right\rangle(Q)\left[\frac{\alpha_{s}(Q)}{\alpha_{s}(\mu)}\right]^{\frac{2}{27}}$ The values of the gluon condensate and three-gluon condensate are also taken to be the standard values $\left\langle\frac{\alpha_{s} G G}{\pi}\right\rangle=0.012 \mathrm{GeV}^{4}$ and $\left\langle g_{s}^{3} G G G\right\rangle=0.045 \mathrm{GeV}^{6}$ [1].

In the article, we neglect the small $u, d$ quark masses and take the $\overline{M S}$ masses $m_{c}\left(m_{c}\right)=$ $(1.275 \pm 0.025) \mathrm{GeV}, m_{b}\left(m_{b}\right)=(4.18 \pm 0.03) \mathrm{GeV}$ and $m_{s}(\mu=2 \mathrm{GeV})=(0.095 \pm 0.005) \mathrm{GeV}$ from the Particle Data Group [2], and take into account the energy-scale dependence of the $\overline{M S}$ masses from the renormalization group equation,

$$
\begin{aligned}
& m_{s}(\mu)=m_{s}(2 \mathrm{GeV})\left[\frac{\alpha_{s}(\mu)}{\alpha_{s}(2 \mathrm{GeV})}\right]^{\frac{4}{9}}, \\
& m_{c}(\mu)=m_{c}\left(m_{c}\right)\left[\frac{\alpha_{s}(\mu)}{\alpha_{s}\left(m_{c}\right)}\right]^{\frac{12}{25}}, \\
& m_{b}(\mu)=m_{b}\left(m_{b}\right)\left[\frac{\alpha_{s}(\mu)}{\alpha_{s}\left(m_{b}\right)}\right]^{\frac{12}{23}}, \\
& \alpha_{s}(\mu)=\frac{1}{b_{0} t}\left[1-\frac{b_{1}}{b_{0}^{2}} \frac{\log t}{t}+\frac{b_{1}^{2}\left(\log ^{2} t-\log t-1\right)+b_{0} b_{2}}{b_{0}^{4} t^{2}}\right],
\end{aligned}
$$

where $t=\log \frac{\mu^{2}}{\Lambda^{2}}, b_{0}=\frac{33-2 n_{f}}{12 \pi}, b_{1}=\frac{153-19 n_{f}}{24 \pi^{2}}, b_{2}=\frac{2857-\frac{5033}{9} n_{f}+\frac{325}{27} n_{f}^{2}}{128 \pi^{3}}, \Lambda=213 \mathrm{MeV}, 296 \mathrm{MeV}$ and $339 \mathrm{MeV}$ for the flavors $n_{f}=5,4$ and 3, respectively [2]. In calculations, we take $n_{f}=4$ and $\mu_{c(b)}=1(3) \mathrm{GeV}$ for the charmed (bottom) tensor mesons. We choose the energy scales $\mu_{c}$ and $\mu_{b}$ for the charmed mesons and bottom mesons respectively based on the crude estimation, $\mu_{c}=\sqrt{M_{D}^{2}-m_{c}^{2}} \approx \sqrt{1.9^{2}-1.5^{2}} \mathrm{GeV} \approx 1 \mathrm{GeV}, \mu_{b}=\sqrt{M_{B}^{2}-m_{b}^{2}} \approx \sqrt{5.3^{2}-4.5^{2}} \mathrm{GeV} \approx 3 \mathrm{GeV}$, where the $M_{D}$ and $M_{B}$ are the masses of the ground states (of the pseudoscalar mesons), the $m_{c}$ and $m_{b}$ are constituent quark masses. The strong coupling constant $\alpha_{s}(\mu)$ in itself is not a physical observable, but rather a quantity defined in the context of perturbation theory, which enters predictions for experimentally measurable observables. We can extract the value of the $\alpha_{s}(\mu)$ from the experimental data at a special energy scale $\mu$, then fit the parameter $\Lambda$ with the expressions of the $\alpha_{s}(\mu)$ from one-loop, two-loop, three-loop, or four-loop renormalization group equations. The values of the $\alpha_{s}(\mu)$ from three-loop renormalization group equation are already compatible with that from different determination [2, we prefer the expression in Eq.(16), not the crude one-loop approximation.

We impose the two criteria (pole dominance and convergence of the operator product expansion) on the charmed (or bottom) tensor mesons, and search for the optimal values of the Borel parameters. The threshold parameters, Borel parameters, pole contributions and the resulting masses and decay constants are shown explicitly in Table 1. The pole contributions are about $(45-80) \%$ and $(45-65) \%$ for the charmed and bottom tensor mesons, respectively, the pole dominance is well satisfied. On the other hand, the dominant contributions come from the perturbative term, while the total contributions come from the gluon condensate and three-gluon condensates are about $(5-10) \%$ and $(2-5) \%$ for the charmed and bottom tensor mesons, respectively, the operator product expansion is well convergent. The two criteria of the QCD sum rules are fully satisfied, we expect to obtain reasonable predictions. 


\begin{tabular}{|c|c|c|c|c|c|}
\hline \hline & $M^{2}\left(\mathrm{GeV}^{2}\right)$ & $s_{0}\left(\mathrm{GeV}^{2}\right)$ & pole & $M_{T}(\mathrm{GeV})$ & $f_{T}(\mathrm{MeV})$ \\
\hline$D_{2}^{*}(2460)$ & $1.5-2.1$ & $8.5 \pm 0.5$ & $(44-78) \%$ & $2.46 \pm 0.09$ & $182 \pm 20$ \\
\hline$D_{s 2}^{*}(2573)$ & $1.6-2.4$ & $9.5 \pm 0.5$ & $(48-83) \%$ & $2.58 \pm 0.09$ & $222 \pm 21$ \\
\hline$B_{2}^{*}(5747)$ & $4.6-5.4$ & $39 \pm 1$ & $(44-65) \%$ & $5.73 \pm 0.06$ & $110 \pm 11$ \\
\hline$B_{s 2}^{*}(5840)$ & $5.2-6.0$ & $41 \pm 1$ & $(46-64) \%$ & $5.84 \pm 0.06$ & $134 \pm 11$ \\
\hline \hline
\end{tabular}

Table 1: The Borel parameters, continuum threshold parameters, pole contributions, masses and decay constants for the heavy tensor mesons.

In Figs.6-7, we plot the masses and decay constants with variations of the Borel parameters. From the figures, we can see that they are rather stable with variations of the Borel parameters in the Borel windows, it is reliable to extract the masses and decay constants. The predicted masses are in excellent agreement with the experimental data [2]. The predictions $f_{D_{2}^{*}}=(0.182 \pm$ $0.020) \mathrm{GeV}, f_{D_{s 2}^{*}}=(0.222 \pm 0.021) \mathrm{GeV}$ are compatible with the values $f_{D_{2}^{*}}=(0.225 \pm 0.067) \mathrm{GeV}$, $f_{D_{s 2}^{*}}=(0.237 \pm 0.113) \mathrm{GeV}$ from Ref.[14]. In Ref.[14], the contributions of the gluon condensates, three-gluon condensates, four-quark condensates and some mixed condensates are neglected, so the present predictions are more robust. We can take the decay constants as basic input parameters and study the revelent processes with the three-point QCD sum rules or the light-cone QCD sum rules, for example, the strong decays

$$
\begin{aligned}
D_{2}^{*}(2460)^{0} & \rightarrow D^{+} \pi^{-}, D^{*+} \pi^{-}, D^{0} \pi^{0}, D^{* 0} \pi^{0}, \\
D_{s 2}^{*}(2573)^{+} & \rightarrow D^{0} K^{+}, D^{* 0} K^{+}, D^{+} K^{0}, D^{*+} K^{0}, D_{s}^{+} \pi^{0}, D_{s}^{*+} \pi^{0}, \\
B_{2}^{*}(5741)^{0} & \rightarrow B^{+} \pi^{-}, B^{*+} \pi^{-}, B^{0} \pi^{0}, B^{* 0} \pi^{0} \\
B_{s 2}^{*}(5840)^{0} & \rightarrow B^{+} K^{-}, B^{*+} K^{-}, B^{0} \bar{K}^{0}, B^{* 0} \bar{K}^{0}, B_{s}^{0} \pi^{0}, B_{s}^{* 0} \pi^{0} .
\end{aligned}
$$

The central values

$$
\frac{f_{D_{s 2}^{*}}}{f_{D_{2}^{*}}}=\frac{f_{B_{s 2}^{*}}}{f_{B_{2}^{*}}}=1.21
$$

the heavy quark symmetry works well. Furthermore, the $S U(3)$ breaking effects are compatible with the experimental data 24],

$$
\frac{f_{D_{s}}}{f_{D}}=1.258 \pm 0.038
$$

the approximation $\frac{f_{D_{s 2}^{*}}}{f_{D_{2}^{*}}} \approx \frac{f_{B_{s 2}^{*}}^{*}}{f_{B_{2}^{*}}^{*}} \approx \frac{f_{D_{s}}}{f_{D}}$ is reasonable.

If we use the non-covariant currents instead of the covariant currents, the gluon condensate and the three-gluon condensate in Eq.(14) have no contributions, the masses and the decay constants change as

$$
\begin{aligned}
\delta M_{D_{2}^{*}} & =-27 \mathrm{MeV}, \quad \delta f_{D_{2}^{*}}=3 \mathrm{MeV}, \\
\delta M_{D_{s 2}^{*}} & =-20 \mathrm{MeV}, \quad \delta f_{D_{s 2}^{*}}=2 \mathrm{MeV}, \\
\delta M_{B_{2}^{*}} & =-14 \mathrm{MeV}, \quad \delta f_{B_{2}^{*}} \approx 0 \mathrm{MeV}, \\
\delta M_{B_{s 2}^{*}} & =-10 \mathrm{MeV}, \quad \delta f_{B_{s 2}^{*}} \approx 0 \mathrm{MeV} .
\end{aligned}
$$

There are effective cancelations among the contributions of the three-gluon condensate $\left\langle g_{s}^{3} G G G\right\rangle$ from different Feynman diagrams, and among the contributions of the three-gluon condensate $\left\langle g_{s}^{3} G G G\right\rangle$ and the four quark condensate $g_{s}^{2}\langle\bar{q} q\rangle^{2}$. The masses and the decay constants remain 

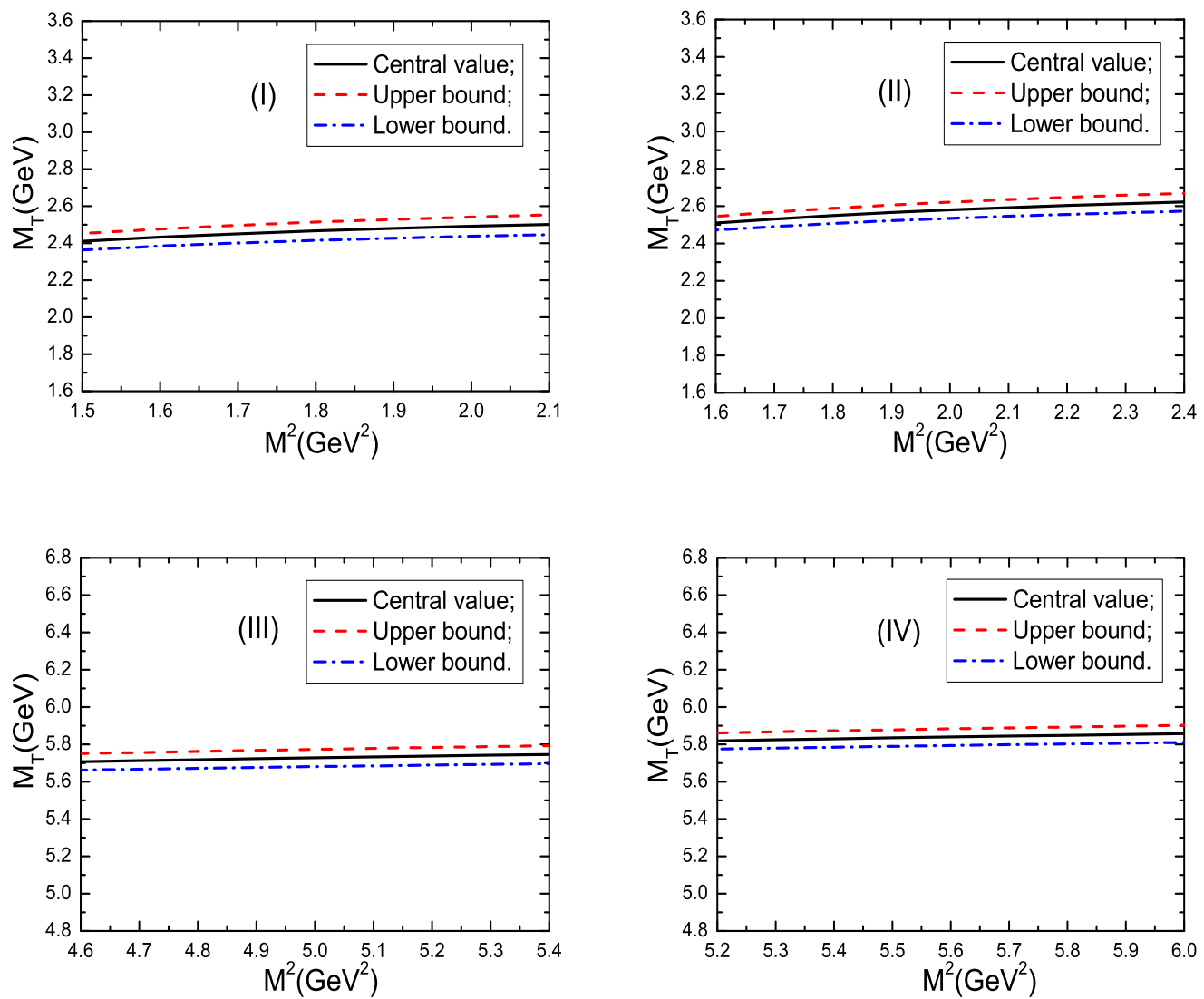

Figure 6: The masses of the tensor mesons with variations of the Borel parameters $M^{2}$, where the (I), (II), (III), (IV) denote the $D_{2}^{*}(2460), D_{s 2}^{*}(2573), B_{2}^{*}(5747), B_{s 2}^{*}(5840)$, respectively. 

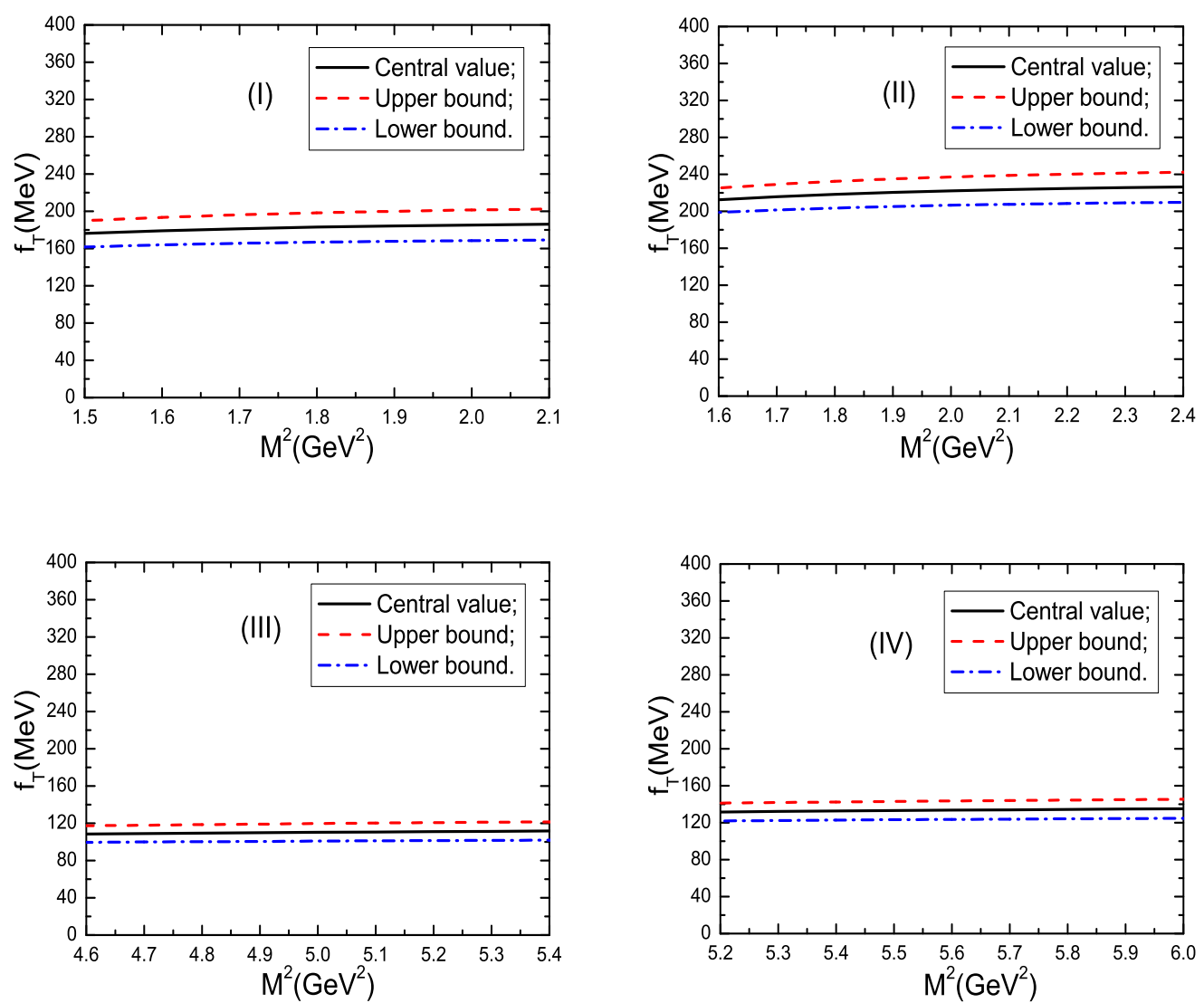

Figure 7: The decay constants of the tensor mesons with variations of the Borel parameters $M^{2}$, where the (I), (II), (III), (IV) denote the $D_{2}^{*}(2460), D_{s 2}^{*}(2573), B_{2}^{*}(5747), B_{s 2}^{*}(5840)$, respectively. 
almost unchanged if the dimension- 6 vacuum condensates are neglected,

$$
\begin{aligned}
\delta M_{D_{2}^{*}} & \approx 0 \mathrm{MeV}, \quad \delta f_{D_{2}^{*}} \approx 0 \mathrm{MeV}, \\
\delta M_{D_{s 2}^{*}} & \approx 0 \mathrm{MeV}, \quad \delta f_{D_{s 2}^{*}} \approx 0 \mathrm{MeV}, \\
\delta M_{B_{2}^{*}} & \approx 0 \mathrm{MeV}, \quad \delta f_{B_{2}^{*}} \approx 0 \mathrm{MeV}, \\
\delta M_{B_{s 2}^{*}} & \approx 0 \mathrm{MeV}, \quad \delta f_{B_{s 2}^{*}} \approx 0 \mathrm{MeV} .
\end{aligned}
$$

In this article, we neglect the perturbative $\alpha_{s}$ corrections. In the massless limit, taking into accounting the perturbative $\alpha_{s}$ corrections amounts to multiplying the perturbative terms by a factor $\left(1-\frac{\alpha_{s}}{\pi}\right)\left[19\right.$. Now, we estimate the perturbative $\alpha_{s}$ contributions by multiplying the perturbative terms by the factor $\left(1-\frac{\alpha_{s}}{\pi}\right)$, which leads to the following changes,

$$
\begin{aligned}
\delta M_{D_{2}^{*}} & \approx 0 \mathrm{MeV}, \quad \delta f_{D_{2}^{*}}=-15 \mathrm{MeV} \\
\delta M_{D_{s 2}^{*}} & \approx 0 \mathrm{MeV}, \quad \delta f_{D_{s 2}^{*}}=-18 \mathrm{MeV}, \\
\delta M_{B_{2}^{*}} & \approx 0 \mathrm{MeV}, \quad \delta f_{B_{2}^{*}}=-5 \mathrm{MeV}, \\
\delta M_{B_{s 2}^{*}} & \approx 0 \mathrm{MeV}, \quad \delta f_{B_{s 2}^{*}}=-6 \mathrm{MeV}
\end{aligned}
$$

In calculations, we observe that the masses decrease monotonously with increase of the energy scales while the decay constants increase monotonously with increase of the energy scales. If we enlarge the energy scales by $\mu_{c(b)} \rightarrow \mu_{c(b)}+300 \mathrm{MeV}$, then

$$
\begin{aligned}
\delta M_{D_{2}^{*}}=-44 \mathrm{MeV}, & \delta f_{D_{2}^{*}}=27 \mathrm{MeV}, \\
\delta M_{D_{s 2}^{*}}=-46 \mathrm{MeV}, & \delta f_{D_{s 2}^{*}}=27 \mathrm{MeV}, \\
\delta M_{B_{2}^{*}}=-37 \mathrm{MeV}, & \delta f_{B_{2}^{*}}=11 \mathrm{MeV}, \\
\delta M_{B_{s 2}^{*}}=-36 \mathrm{MeV}, & \delta f_{B_{s 2}^{*}}=12 \mathrm{MeV},
\end{aligned}
$$

the changes are sizeable, but they are small compared to the energy scale augment $300 \mathrm{MeV}$. The correlation functions $\Pi\left(p^{2}\right)$ can be written as

$$
\Pi\left(p^{2}\right)=\int_{m_{Q}^{2}(\mu)}^{s_{0}} d s \frac{\rho_{Q C D}(s, \mu)}{s-p^{2}}+\int_{s_{0}}^{\infty} d s \frac{\rho_{Q C D}(s, \mu)}{s-p^{2}},
$$

through dispersion relation at the QCD side, and they are scale independent, $\frac{d}{d \mu} \Pi\left(p^{2}\right)=0$, which does not amount to

$$
\frac{d}{d \mu} \int_{m_{Q}^{2}(\mu)}^{s_{0}} d s \frac{\rho_{Q C D}(s, \mu)}{s-p^{2}} \rightarrow 0,
$$

as the perturbative corrections to all orders are neglected and truncations $s_{0}$ set in. The correlation between the threshold $m_{Q}^{2}(\mu)$ and continuum threshold $s_{0}$ is unknown. We cannot obtain energy scale independent QCD sum rules, but we can choose the reasonable energy scales based on some theoretical analysis.

\section{Conclusion}

In this article, we calculate the contributions of the vacuum condensates up to dimension- 6 in the operator product expansion, study the masses and decay constants of the heavy tensor mesons using the QCD sum rules. The predicted masses of the $D_{2}^{*}(2460), D_{s 2}^{*}(2573), B_{2}^{*}(5747), B_{s 2}^{*}(5840)$ are in excellent agreement with the experimental data, while the ratios of the decay constants $\left.\frac{f_{D_{s 2}^{*}}}{f_{D_{2}^{*}}^{*}} \approx \frac{f_{B_{s}^{*}}^{*}}{f_{B_{2}^{*}}^{*}} \approx \frac{f_{D_{s}}}{f_{D}}\right|_{\exp }$, where the exp denotes the experimental value. The decay constants can be taken as basic input parameters in studying the strong decays with the three-point QCD sum rules or the light-cone QCD sum rules. 


\section{Acknowledgements}

This work is supported by National Natural Science Foundation, Grant Numbers 11375063, the Fundamental Research Funds for the Central Universities, and Natural Science Foundation of Hebei province, Grant Number A2014502017.

\section{References}

[1] E. S. Swanson, Phys. Rept. 429 (2006) 243; S. Godfrey and S. L. Olsen, Ann. Rev. Nucl. Part. Sci. 58 (2008) 51; M. B. Voloshin, Prog. Part. Nucl. Phys. 61 (2008) 455; N. Drenska, R. Faccini, F. Piccinini, A. Polosa, F. Renga and C. Sabelli, Riv. Nuovo Cim. 033 (2010) 633; N. Brambilla et al, Eur. Phys. J. C71 (2011) 1534.

[2] J. Beringer et al, Phys. Rev. D86 (2012) 010001.

[3] V. M. Abazov et al, Phys. Rev. Lett. 99 (2007) 172001.

[4] T. Aaltonen et al, Phys. Rev. Lett. 102 (2009) 102003.

[5] T. Aaltonen et al, Phys. Rev. Lett. 100 (2008) 082001.

[6] V. Abazov et al, Phys. Rev. Lett. 100 (2008) 082002.

[7] R. Aaij et al, Phys. Rev. Lett. 110 (2013) 151803.

[8] M. A. Shifman, A. I. Vainshtein and V. I. Zakharov, Nucl. Phys. B147 (1979) 385; Nucl. Phys. B147 (1979) 448.

[9] L. J. Reinders, H. Rubinstein and S. Yazaki, Phys. Rept. 127 (1985) 1.

[10] S. Narison, Camb. Monogr. Part. Phys. Nucl. Phys. Cosmol. 17 (2002) 1.

[11] P. Colangelo and A. Khodjamirian, hep-ph/0010175.

[12] Z. G. Wang, JHEP 1310 (2013) 208; P. Gelhausen, A. Khodjamirian, A. A. Pivovarov and D. Rosenthal, Phys. Rev. D88 (2013) 014015; M. J. Baker, J. Bordes, C. A. Dominguez, J. Penarrocha and K. Schilcher, JHEP 1407 (2014) 032; W. Lucha, D. Melikhov and S. Simula, Phys. Lett. B735 (2014) 12; S. Narison, arXiv:1404.6642,

[13] S. Narison, Phys. Lett. B605 (2005) 319; Z. G. Wang, Phys. Rev. D73 (2006) 094020; Z. G. Wang and S. L. Wan, Phys. Rev. D74 (2006) 014017; M. Nielsen, Phys. Lett. B634 (2006) 35; Z. G. Wang, J. Phys. G34 (2007) 753; Y. B. Dai, X. Q. Li, S. L. Zhu and Y. B. Zuo, Eur. Phys. J. C55 (2008) 249; Z. G. Wang, Chin. Phys. Lett. 25 (2008) 3908.

[14] H. Sundu and K. Azizi, Eur. Phys. J. A48 (2012) 81; K. Azizi, H. Sundu, J. Y. Sungu and N. Yinelek, Phys. Rev. D88 (2013) 036005.

[15] K. Azizi, H. Sundu, A. Turkan and E. V. Veliev, J. Phys. G41 (2014) 035003; K. Azizi, Y. Sarac and H. Sundu, arXiv:1402.6887.

[16] M. Neubert, Phys. Rept. 245 (1994) 259.

[17] Y. B. Dai, C. S. Huang, M. Q. Huang and C Liu, Phys. Lett. B390 (1997) 350; Y. B. Dai, C. S. Huang, C. Liu and S. L. Zhu, Phys. Rev. D68 (2003) 114011.

[18] T. M. Aliev and M. A. Shifman, Phys. Lett. B112 (1982) 401.

[19] L. J. Reinders, S. Yazaki and H. R. Rubinstein, Nucl. Phys. B196 (1982) 125. 
[20] E. Bagan and S. Narison, Phys. Lett. B214 (1988) 451.

[21] T. M. Aliev, K. Azizi and V. Bashiry, J. Phys. G37 (2010) 025001; T. M. Aliev, K. Azizi and M. Savci, J. Phys. G37 (2010) 075008; H. Y. Cheng, Y. Koike and K. C. Yang, Phys. Rev. D82 (2010) 054019; F. K. Guo, B. Kubis and A. Wirzba, Phys. Rev. D85 (2012) 014014.

[22] J. J. Zhu and M. L. Yan, hep-ph/9903349; and references therein.

[23] P. Pascual and R. Tarrach, Lect. Notes Phys. 194 (1984) 1; and references therein.

[24] J. L. Rosner and S. Stone, arXiv:1309.1924. 